\title{
RAPID PACING INDUCES FRACTIONATION OF THE ACTIVATION WAVEFRONT INTO MULTIPLE WAVELETS
}

\author{
Hofer $\mathrm{E}^{1}$, Sanchez-Quintana $\mathrm{D}^{2}$, Arnold $\mathrm{R}^{1}$ \\ ${ }^{1}$ Institute of Biophysics, Medical University Graz, Austria \\ ${ }^{2}$ Depart. de Anatomia y Biologia Cellular, Universidad de Extremadura, Badajoz, Spain \\ ernst.hofer@medunigraz.at
}

\begin{abstract}
Atrial flutter and atrial fibrillation can be caused by heterogeneities in membrane function as well as in macro- and microstructure. Rapid pacing techniques combined with potential recording techniques can help to localize critical arrhythmogenic substrates within the atrium. This work describes the heterogeneity of local conduction in the cavotricuspid isthmus (CTI) during electrophysiological experiments with Rabbit hearts. By means of rapid pacing techniques it can be shown, that local blockade of excitation spread can be predicted using appropriate pacing protocols. The pre-block behaviour is characterized by a substantial increase in the degree of fractionation and in a decrease of amplitude in extracellular potentials.
\end{abstract}

Keywords: atrial flutter, rapid pacing, complex fractionated atrial electrogram, electrophysiological experiment

\section{Introduction}

Atrial fibrillation and atrial flutter is often preceded by tachycardia. Hence rapid pacing can be used to study the behaviour of cardiac activation before local or global block of excitation spread arises. Increased pacing rate leads to changes in action potential duration and alters the excitation spread. The smooth wave front of depolarization then may break into multiple wavelets with small delays to each other and following complex pathways. These effects are caused by discontinuities in the macroand microstructure of cardiac tissue. We postulate that during rapid pacing, these effects would lead to increased signal fractionation and spatial heterogeneity, specifically when pacing intervals come close to the absolute refractory period. In electrophysiological experiments with right atria from Rabbits we studied the region of the cavotricuspid isthmus (CTI). Clinical electrophysiologists see this region as one of the targets for catheter ablation to cure atrial arrhythmias [1]. Specific pacing protocols were applied to induce arrhythmogenic conditions. Spatial heterogeneity and beat-to-beat changes of local conduction were analyzed by means of multisite recording techniques of extracellular potentials with ultra-high spatial and temporal resolution [2].

\section{Methods}

Electrophysiological experiments were carried out in accordance to the national ethic guidelines. Right atrial specimen were dissected from isolated Rabbit hearts and placed in a tissue bath with oxygenated Tyrode's solution at $36^{\circ} \mathrm{C}$. Current pulses of $1 \mathrm{~ms}$ duration and twice the threshold level were applied by means of a $50 \mu \mathrm{m}$ thick tungsten wire at given sites of the tissue. A ramp-like pacing protocol was executed i.e. subsequent stimuli were applied with decreased pacing cycle length (PCL) compared to the previous one until conduction block occurred. Multisite recordings of extracellular signals $\Phi_{e}(t)$ of the last 6 beats before block were used to analyze the quality of local conduction of the depolarization wave. Amplitude of the signals as well as the fractionation index (FI), i.e. the number of negative deflections of $d \Phi_{e}(t) / d t$ [3] were used to describe the increasingly hampered excitation process. At least one of the four-electrode-sensors was placed at sites were muscle bundles merge or branch, i.e. at sites of structural discontinuities.

\section{Results}
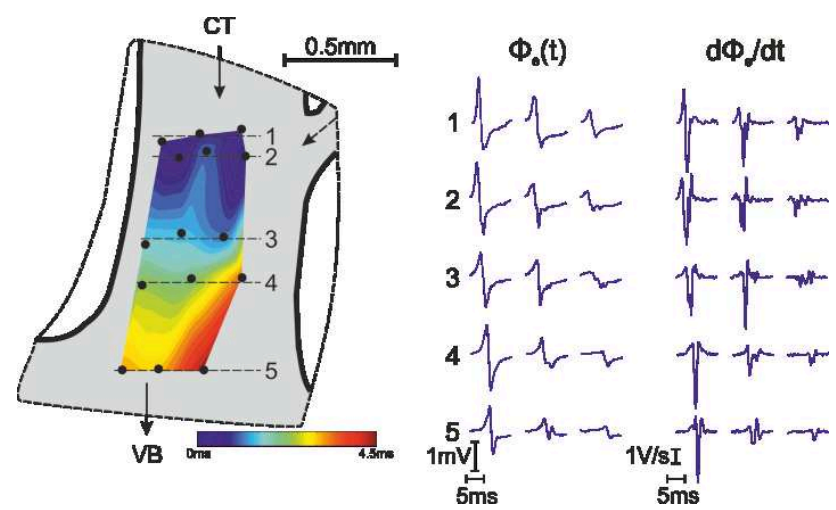

Figure 1: Spatial heterogeneity of depolarization signals within one PectM. Recordings on the right in line 2 and 3 indicate incoming wavelet from the top right branch.

Along the excitation pathway in the CTI, groups of pectinate muscles (PectM) connect the Crista Terminalis (CT) with the vestibule (VB). PectMs branch into two or three fibres and merge again before reaching the VB. At these sites complex signal waveforms with multiple deflections in $d \Phi_{e}(t) / d t$ can be expected frequently, specifically during rapid pacing close to the block interval. An example of spatial heterogeneity of local signals is depicted in Fig. 1. A map of signals in the lower part of a PectM is shown. Note the small amplitudes and the high degree of fractionation of the signals taken at the right edge of the mapping area. This indicates an electrically uncoupled wavelet entering from the small merging fibre from top right. 
Diversity of depolarization signals is not only present in terms of spatial heterogeneity but also at one given recording site in response to subsequent pacing stimuli when PCL decreases. During control values of PCL $\Phi_{e}(t)$ and $d \Phi_{e}(t) / d t$ are quite uniform showing a large amplitude and just minor distortion visible as a small secondary deflection in $d \Phi_{e}(t) / d t$ (see Fig. 2). During the last 6 stimuli before block arises amplitude diminishes and the fractionation index FI increases progressively.
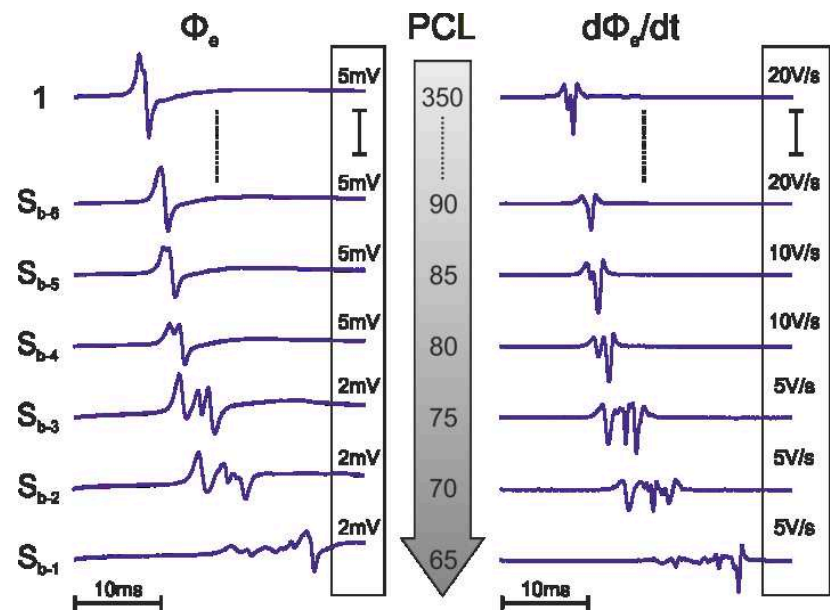

Figure 2: Progressive change of $\Phi_{e}(t)$ and $d \Phi_{e}(t) / d t$ with PCL approaching absolute refractory period. Ramp like pacing protocol was used.

We analysed a large number of recordings taken in CT (239), PectM (1037) and VB (264) from 15 hearts. The distribution of FI within each class of tissue differs markedly (see Tab. 1) due to different histological structure. CT represents the most complex microstructure and pathways for the cardiac impulse entering from the sinus node area into the CT resulting in the lowest portion of uniform signals with $\mathrm{FI}=1$. In the class of PectM signals the dominant portion of recordings is uniform. In the VB we found a higher number of uniform signals compared to CT but also a much larger portion of large FI signals $(\mathrm{FI}=8-10)$. This might be linked to the fact, that on one hand very compact strands of PectM fibres incorporate smoothly in the $\mathrm{VB}$ and on the other hand signals are colliding there with crossing fibres.

Table 1: Fractionation Index distribution obtained in CT, PectM and VB (given in \%).

\begin{tabular}{llll}
\hline FI & CT & PectM & VB \\
\hline 1 & 20,51 & 49,03 & 46,89 \\
2 & 26,50 & 26,56 & 17,84 \\
3 & 17,95 & 11,19 & 14,11 \\
4 & 15,81 & 5,45 & 5,39 \\
5 & 8,12 & 3,60 & 5,81 \\
$6-10$ & 11,11 & 4,18 & 9,96 \\
\hline
\end{tabular}

\section{Discussion}

Uniform conduction with biphasic extracellular signals along cable like strands of cardiac fibres can be predicted by computer simulation. Within a network of such strands at least piecewise continuous conduction can be expected interrupted just at branching and merging sites representing macroscopic discontinuities. At microscopic scale layers of connective tissue are oriented parallel to the muscle fibres and form obstacles changing the signal waveform with directional effects [4]. By such microstructures the depolarization wave front breaks up into wavelets with delays in the range from 0.5 to $10 \mathrm{~ms}$. It should be emphasized that this type of fractionation is different to those seen in clinical human atrial electrograms with multiple deflections in the range of 50$100 \mathrm{~ms}$. Fractionations like demonstrated here with ultrahigh resolution systems (50 $\mu \mathrm{m}$ inter-electrode distances) would not be detectable with relatively coarse recording systems (mm range) like used in clinical electrophysiology.

At a microscopic size scale the CTI can be seen as a network of piecewise cable-like muscle structures producing spatial heterogeneous signals waveforms. In addition to this macro- and microstructure related heterogeneity, temporal parameters like rapid pacing can change the character of local conduction from uniform towards complex and lead to local block and atrial arrhythmia [5]. High resolution recording systems could help to elucidate the mechanisms of complex conduction and improve analyzing techniques to identify zones for ablation.

\section{Acknowledgement}

This work has been supported by the Austrian Science Fund (FWF) grant P19993-N15 and grant F3210-N18.

\section{Bibliography}

[1] J.A .Cabrera, D. Sanchez-Quintana, J. Farre et al.. "The inferior right atrial isthmus: further architectural insights for current and coming ablation technologies", J. Cardiovasc. Electrophysiol., Vol.16, pp. 402-408, 2005.

[2] E. Hofer, F. Keplinger, T. Thurner, T. Wiener, D. Sanchez-Quintana, V. Climent, Plank G. "A new floating sensor array to detect electric near fields of beating heart preparations". Biosens Bioelectron., vol. 21(12), pp. 2232-2239, June 2006

[3] T. Wiener, F.O. Campos, G. Plank, E. Hofer. "Decomposition of fractionated local electrograms using an analytic signal model based on sigmoid functions", Biomed Tech, pp.1-12, Oct 2012.

[4] F. Campos, T. Wiener, A. Prassl, R. Weber Dos Santos, D. Sanchez-Quintana, H. Ahammer, G. Plank, E. Hofer. "Electro-Anatomical Characterization of Atrial Microfibrosis in a Histologically Detailed Computer Model". IEEE Trans Biomed Eng. 2013 Apr 3 (Epub ahead of print).

[5] R. Arnold and E. Hofer, "Pre-block behavior of intra and extracellular parameters during different pacing protocols - preliminary results," in World Congress on Medical Physics and Biomedical Engineering May 26-31, 2012, Beijing, China, vol. 39, pp. 395$397,2012$. 